Artigo Original

Original Article

\title{
Perfil da fala de pacientes submetidos à palatoplastia primária
}

\author{
Speech profile of patients undergoing primary \\ palatoplasty
}

Descritores

Fissura Palatina

Fala

Cirurgia

Insuficiência Velofaríngea

Fonoaudiologia

Keywords

Cleft Palate

Speech

Surgery

Velopharyngeal Insufficiency

Speech, Language and Hearing

Sciences

Endereço para correspondência:

Claudia Regina Furquim de Andrade

Rua Cipotânea, 51, Cidade

Universitária, São Paulo (SP), Brasil,

CEP: 05360-160.

E-mail: clauan@usp.br

Recebido em: Setembro 02, 2016

\section{RESUMO}

Objetivo: caracterizar o perfil e a fala de pacientes submetidos à palatoplastia primária em um hospital escola de São Paulo, levando-se em consideração a idade do paciente no momento da cirurgia (precoce até os 2 anos de idade e tardio após 2 anos). Método: 97 indivíduos, de ambos os gêneros, com diagnóstico de fissura de palato associada ou não à de lábio, divididos em dois grupos: 1) grupo precoce (GP), composto por 43 indivíduos operados até o segundo ano de vida; 2) grupo tardio (GT), composto por 54 indivíduos operados após o segundo ano. Os participantes foram submetidos à avaliação clínica fonoaudiológica. Os parâmetros avaliados e considerados para o estudo foram: classificação da ressonância, presença de ronco nasal audível, ocorrência de fraca pressão intraoral, ocorrência de emissão nasal, classificação da inteligibilidade de fala e presença de distúrbios articulatórios compensatórios (DACs). Uma porcentagem randomicamente selecionada de participantes (30\%) foi reavaliada por mais duas fonoaudiólogas e a comparação entre os juízes indicou alta concordância. Resultados: (nível de significância de 5\%): os grupos não se diferenciaram em relação à classificação da ressonância $(p=0,067)$, grau de hipernasalidade $(p=0,113)$, presença de ronco nasal $(p=0,179)$, ocorrência de fraca pressão intraoral $(\mathrm{p}=0,152)$, ocorrência de emissão nasal $(\mathrm{p}=0,369)$ e classificação da inteligibilidade de fala $(\mathrm{p}=0,113)$. Em relação à presença de $\mathrm{DACs}$, os grupos se diferenciaram $(\mathrm{p}=0,020)$, com maior ocorrência de fonemas alterados no GT. Conclusão: foi possível caracterizar o perfil geral e de fala dos pacientes submetidos à palatoplastia primária do referido hospital escola. Concluiu-se que a realização da cirurgia precocemente traz melhores resultados em relação à fala.

Trabalho realizado no Departamento de Fisioterapia, Fonoaudiologia e Terapia Ocupacional da Faculdade de Medicina, Universidade de São Paulo - USP - São Paulo (SP), Brasil.

${ }^{1}$ Universidade de São Paulo - USP - São Paulo (SP), Brasil.

${ }^{2}$ Universidade de Brasília - UnB - Brasília (DF), Brasil.

Fonte de financiamento: nada a declarar

Conflitos de interesses: nada a declarar. 


\section{INTRODUÇÃO}

As fissuras labiopalatinas representam a anomalia congênita de face mais comum, acometendo lábio e/ou palato, e podem ocorrer de forma isolada ou associada a outras malformações complexas, trazendo consequências estéticas e funcionais que requerem reabilitação ao longo da vida ${ }^{(1,2)}$. São as anomalias congênitas mais frequentes tratadas em centros de cirurgia craniofacial, apresentando uma incidência de 1 para cada 600 nascidos vivos ${ }^{(3)}$. Diversas alterações anatômicas e funcionais são observadas como consequência da fissura labiopalatina, ocasionando alterações em relação ao crescimento do terço médio da face, à audição e à funcionalidade do esfíncter velofaríngeo. Esta última interfere diretamente nas funções de sucção, deglutição e fala ${ }^{(4)}$.

Um dos principais objetivos do fechamento da fissura palatina é estabelecer uma adequada função velofaríngea, para que o desenvolvimento da comunicação ocorra da forma mais adequada possível. Quando ocorre o fechamento tardio, as chances de desenvolvimento de fala adequado diminuem significativamente ${ }^{(5-7)}$. Estudo anterior ${ }^{(6)}$ mostrou que existe diferença estatisticamente significante entre indivíduos operados precocemente e tardiamente em relação à presença de processos fonológicos, sendo notado que estes aparecem com menos frequência no grupo que realizou cirurgia precocemente.

A falha no fechamento velofaríngeo, seja em decorrência da presença da fissura ou de disfunção velofaríngea, no que se refere aos aspectos relacionados à fala, tem como consequência a perda de parte da corrente aérea para a cavidade nasal levando ao aparecimento de sintomas que podem prejudicar a inteligibilidade da fala de forma direta ou indireta ${ }^{(8)}$. A hipernasalidade, a emissão de ar nasal e a fraca pressão aérea intraoral são consideradas consequências diretas ou primárias ${ }^{(6,8)}$. Como consequências indiretas ou secundárias, define-se a presença de distúrbios articulatórios compensatórios (DACs), estratégias utilizadas pelo indivíduo com o objetivo de compensar em outros pontos do trato vocal a incapacidade de impor pressão na cavidade oral ${ }^{(6,8)}$.

Durante a adequada funcionalidade da velofaringe, é necessário que o palato mole, as paredes laterais e posterior da faringe realizem um movimento amplo e sincronizado, permitindo a manutenção do toque entre estas estruturas e assegurando a total separação entre as cavidades oral e nasal durante a produção dos sons orais ${ }^{(9)}$. A inadequação desta funcionalidade faz com que os bebês com fissura labiopalatina, mesmo nas primeiras tentativas de vocalização no segundo e terceiro meses de vida, as realize de forma diferente em comparação aos bebês sem fissura. Aos 6 meses de idade, uma criança começa a produzir suas primeiras consoantes anteriores, e, no caso de crianças com fissura, pode haver dificuldades na execução dessa tarefa, o que originaria articulações glóticas ou faríngeas $^{(10)}$.

O primeiro ano da criança é de suma importância para a aquisição da fala e da linguagem ${ }^{(10)}$. Na literatura, é bem estabelecido que o reparo cirúrgico da fissura palatina deve ser realizado precocemente, antes de dois anos de idade $^{(5,6,11)}$, principalmente considerando a questão da fala ${ }^{(5,12)}$. O tratamento cirúrgico tem por objetivo propiciar a adequada função velofaríngea, essencial na primeira infância ${ }^{(6)}$. Para tal, diferentes técnicas de palatoplastia foram desenvolvidas ao longo dos anos com o objetivo de alcançar um comprimento adequado do palato, de tal modo a permitir o fechamento velofaríngeo. Outro aspecto abordado é a reorientação das fibras musculares do palato, criando uma cinta muscular que permitirá melhor mobilidade do palato mole $\mathrm{e}^{(5)}$.

Entretanto, apesar do avanço na área, ainda é difícil predizer a eficiência do funcionamento muscular para a fala após a cirurgia de palato $^{(13,14)}$. Embora haja o reconhecido esforço para estabelecer a função velofaríngea adequada, estudo realizado por meio da ressonância magnética e reconstrução computadorizada, indicou que até $35 \%$ dos indivíduos com fissura palatina podem permanecer com insuficiência velofaríngea ${ }^{(13)}$. Esta insuficiência pode ser decorrente da dissecção inadequada da musculatura palatina, do comprimento insuficiente do palato ou, ainda, de alterações anatômicas da parede posterior da faringe ${ }^{(13)}$. Outro estudo apresenta que por volta de $30 \%$ dos pacientes podem permanecer com alterações na articulação e ressonância da fala ${ }^{(14)}$ após a palatoplastia.

No Brasil, assim como em outros países em desenvolvimento, nem sempre é possível seguir as etapas do tratamento das fissuras labiopalatinas preconizadas e realizadas pela maioria dos centros internacionais e nacionais, como citato anteriormente, uma vez que, devido a fatores socioeconômicos-culturais, comumente a busca pelo tratamento ocorre com a criança em idade posterior ao período de aquisição da linguagem, muitas vezes já em idade $\operatorname{adulta}^{(2,15,16)}$.

As alterações de fala associadas à disfunção velofaríngea seriam identificadas por meio da avaliação clínica da fala realizada pelo fonoaudiólogo e também por meio da avaliação instrumental da funcionalidade da velofaringe. Os resultados destas avaliações possibilitam a determinação de melhor tratamento e prognóstico ao caso $^{(17)}$.

A avaliação clínica perceptiva da fala é considerada o principal indicador da disfunção velofaríngea, sendo parte essencial no diagnóstico clínico ${ }^{(18)}$. Essa avaliação permite a identificação de alterações, a mensuração da gravidade e a avaliação da efetividade dos tratamentos realizados, ainda que subjetivamente $^{(18,19)}$. Ao longo dos anos, a literatura ${ }^{(19)}$ vem preocupando-se em aperfeiçoar a avaliação perceptivo-auditiva a fim de torná-la menos suscetível a erros decorrentes de sua subjetividade. Assim, sugere-se a adoção de critérios de escores para representar o julgamento do fonoaudiólogo. Dentre as diferentes escalas desenvolvidas para graduar a ressonância e demais características da fala, a mais popular é a escala com intervalos iguais, com a qual o avaliador atribui uma nota ao aspecto analisado, em uma escala linear, na qual o menor número refere-se à ausência de alterações e o maior, ao grau máximo de alteração. Assim, em uma escala de seis pontos na avaliação da hipernasalidade, a categoria um indica a ausência de hipernasalidade e a categoria seis indica o grau mais grave ${ }^{(20)}$.

Dois estudos nacionais ${ }^{(6,21)}$ verificaram a relação entre as alterações de fala e a idade da realização da palatoplastia, considerando-se, para ambos, a melhor idade para a realização

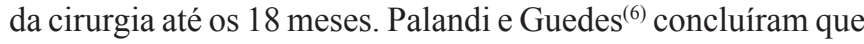
indivíduos que realizaram cirurgia tardiamente apresentaram diferença estatisticamente significante em relação à presença de alguns processos fonológicos, e consideraram que a idade em que se realiza a cirurgia reparadora é fator interveniente para a fala dos indivíduos com fissura. Os resultados de Mituuti et al. ${ }^{(21)}$ apontam que poucos indivíduos apresentam alterações de fala 
após palatoplastia (variando de no mínimo $8 \%$, com fraca pressão aérea intra-oral, ao máximo de $26 \%$ com DACs).

Diante do apresentado, o objetivo deste estudo é caracterizar o perfil e a fala dos pacientes submetidos à palatoplastia primária em um hospital escola de referência da cidade de São Paulo, levando-se em consideração a idade do paciente no momento da cirurgia (momento precoce até os 2 anos de idade, e momento tardio após 2 anos de idade). Trata-se de um centro de referência nacional para casos de fissura labiopalatina, que recebe um grande número de casos novos anualmente. Alguns desses pacientes iniciam seu tratamento na instituição, e outros escolhem o hospital para dar continuidade ao seu atendimento. Devido à grande demanda, identificou-se a necessidade de caracterizar as alterações de fala dos pacientes, independentemente do momento da realização do procedimento cirúrgico para a correção das fissuras, no intuito de se iniciar discussão e proposição de melhorias de atendimento a esses indivíduos.

\section{MÉTODO}

Os procedimentos de seleção e avaliação dos participantes só foram iniciados após os processos éticos pertinentes: parecer da comissão de ética da instituição (CAPPesq HCFMUSP 513.535) e assinatura do Termo de Consentimento Livre e Esclarecido (TCLE) pelos participantes da pesquisa ou responsáveis. Trata-se de estudo transversal observacional.

Participaram do estudo 97 indivíduos com diagnóstico de fissura palatina não sindômica associada ou não à fissura de lábio, encaminhados para avaliação fonoaudiológica entre maio de 2011 e julho de 2015, conforme demanda do serviço. Estes foram divididos em dois grupos: o grupo precoce (GP) e grupo tardio (GT). O GP foi composto por indivíduos que realizaram a palatoplastia primária até o segundo ano de vida, e o GT foi composto por indivíduos que realizaram a palatoplastia primária tardiamente, após os dois anos de idade ${ }^{(6)}$.

O diagnóstico e acompanhamento médico dos participantes foram realizados pela Equipe de Cirurgia Craniomaxilofacial da Divisão de Cirurgia Plástica e Queimaduras do hospital, conforme parceria com esta divisão, que evolve a atuação de cirurgiões plásticos e otorrinolaringologistas.

Foram incluídos no estudo indivíduos: 1) de ambos os gêneros; 2) com idade mínima de seis anos, sem restrição de idade máxima; 3) com diagnóstico de fissura de palato não sindrômica associada ou não à fissura de lábio; 4) corrigida cirurgicamente há no mínimo três meses em relação à data da avaliação fonoaudiológica; e 5) que não houvessem realizado terapia fonoaudiológica prévia e/ou tratamento com próteses.

A idade mínima estabelecida para este estudo baseia-se nos estudos de Andrade et al. ${ }^{(22)}$, que defendem que os processos fonológicos e o desenvolvimento da linguagem oral da criança encontram-se, nesta idade, já finalizados. A utilização deste critério objetiva a exclusão da variável aquisição de linguagem neste estudo.

Foram considerados critérios de exclusão: 1) realização de cirurgias secundárias em palato; 2) presença de alterações neurológicas; 3) histórico de traumas de face; 4) presença de doenças respiratórias; 5) presença de fístulas de palato de qualquer extensão; 6) presença de comorbidades fonoaudiológicas (queixas e déficits de linguagem e auditivos); 7) presença de comprometimento cognitivo ou de nível de consciência que impossibilitasse a compreensão de informações verbais solicitadas para a avaliação e tratamento fonoaudiológicos.

Como citado anteriormente, os participantes haviam sido operados ou não na instituição. Desta forma, não foi possível controlar as variáveis tipo de procedimento cirúrgico e/ou quantidade de cirurgiões envolvidos nestes procedimentos.

Os participantes foram submetidos à rotina de avaliação fonoaudiológica na Unidade de Fonoaudiologia do hospital. Todas as avaliações foram realizadas por um fonoaudiólogo treinado e com experiência na área, pertencente ao grupo de fonoaudiólogos responsáveis pelos atendimentos do Ambulatório de Fissuras Labiopalatinas.

Os dados utilizados para este estudo compõem a gama de aspectos avaliados durante a avaliação de rotina, mais especificamente da Avaliação Perceptivo auditiva da Fala. Para a execução desta avaliação, um protocolo próprio da unidade é aplicado. Este foi elaborado pelos fonoaudiólogos do serviço de Fonoaudiologia, após extensa revisão da literatura e participação em reuniões de classe que visam à padronização de protocolos de avaliação para pacientes fissurados. Este protocolo compreende a avaliação dos aspectos morfológicos do sistema motor oral, avaliação da oclusão e avaliação da fala. Esta última é avaliada por meio da análise de amostras de fala, compostas por trechos de fala dirigida (repetição de vocábulos e frases) com recorrência do som alvo a ser analisado, contendo todos os fonemas do português brasileiro; fala automática durante a contagem de 1 a 10 e, por fim, fala espontânea, solicitando ao paciente a narração de algum acontecimento cotidiano. A verificação da ocorrência ou não do escape de ar nasal durante a fala é realizada por meio do teste de emissão nasal, com o auxílio do espelho de Glatzel.

As amostras são coletadas em sala de terapia fonoaudiológica, com ambiente tratado e específico para este fim. Os participantes permanecem sentados, com um microfone do tipo unidirecional, posicionado a uma distância padronizada de dez centímetros da boca e a um ângulo de $45^{\circ}$. Os estímulos captados pelo microfone são digitalizados pelo software Praat, em extensão .wav, utilizando-se $22.050 \mathrm{~Hz}$ de frequência de amostragem e resolução de 16 bits.

Dentre os parâmetros avaliados pelo protocolo, encontram-se a presença de alterações de ressonância, o grau de alteração da ressonância, a presença de DACs, a presença de ronco nasal, a presença de fraca pressão intraoral, a presença de emissão de ar e o grau de inteligibilidade da fala, variáveis consideradas para este estudo.

As amostras foram analisadas seguindo-se os seguintes critérios: 1) presença ou ausência de hipernasalidade; quando presente, classificação do grau de prejuízo - leve, moderado ou grave; 2) presença ou ausência de hiponasalidade; quando presente, classificação do grau de prejuízo - leve, moderado ou grave; 3) presença ou ausência de DACs; 4) determinação dos tipos de DACs presentes e o número de fonemas em que ocorreram; 5) presença de ronco nasal; 6) presença de fraca pressão intraoral; 6) presença de emissão de ar, audível ou não; 
7) grau de inteligibilidade da fala - adequada, prejuízo leve, prejuízo moderado ou prejuízo grave.

A hipernasalidade foi considerada presente quando verificado excesso de ressonância nasal durante a produção de sons orais da fala ${ }^{(8)} \mathrm{em}$ pelo menos um fonema de cada uma das amostras analisadas. A hiponasalidade foi identificada quando se verificou pouca ressonância nasal durante a produção de sons nasais da fala, em pelo menos um fonema de cada uma das amostras analisadas. A emissão de ar nasal foi determinada quando houve escape do fluxo aéreo para a cavidade nasal durante a produção da fala, podendo ser de forma audível ou não ${ }^{(8)}$, em pelo menos um fonema de cada uma das amostras analisadas.

Os DACs são chamados de compensatórios por ser uma tentativa de compensação diante da dificuldade da produção do fonema no ponto articulatório adequado. Eles são considerados distúrbios do aprendizado, relacionados com as alterações estruturais, principalmente com a presença da fraca pressão intraoral para a produção de consoantes plosivas, fricativas e africadas. A presença dos DACs foi considerada neste estudo quando se verificou a substituição dos sons articulados oralmente por sons articulados em pontos aquém do local da deficiência, na tentativa de aproximar o resultado acústico o mais próximo possível do que se considera como o som normal ${ }^{(23)}$, em pelo menos um fonema da fala dirigida do participante. Os principais DACs são o golpe de glote, a plosiva faríngea, a plosiva dorso médio palatal, a fricativa faríngea, a fricativa velar, a fricativa nasal e a fricativa nasal posterior ${ }^{(24)}$.

A presença de ronco nasal foi definida pela resultante do atrito do ar entre o palato mole e as paredes da faringe, devido ao não fechamento adequado da velofaringe ${ }^{(24)}$, em pelo menos um fonema de cada uma das amostras analisadas. Considerou-se presença de fraca pressão aérea intraoral a emissão com redução da pressão aérea mantida na cavidade oral durante a produção dos sons orais da fala ${ }^{(19)}$, em pelo menos um fonema de cada uma das amostras analisadas. $\mathrm{O}$ escape de ar nasal foi considerado presente quando se observou o escape do fluxo aéreo para a cavidade nasal durante a produção da fala ${ }^{(19)} \mathrm{em}$ pelo menos um fonema de cada uma das amostras analisadas.

Para a análise da inteligibilidade de fala, foram levadas em consideração todas as amostras de fala dos participantes - fala dirigida, fala automática e principalmente a fala espontânea.

\section{Confiabilidade entre as fonoaudiólogas responsáveis pelos atendimentos do Ambulatório de Fissuras Labiopalatinas}

Todos os fonoaudiólogos responsáveis pelos atendimentos do Ambulatório de Fissuras Labiopalatinas passaram por treinamento específico no Serviço de Fonoaudiologia do hospital e só iniciaram suas atividades práticas após atingirem um grau de concordância alta junto aos seus pares.

No entanto, para garantir a confiabilidade do estudo, uma porcentagem randomicamente selecionada de participantes $(30 \%, n=31)$ foi reavaliada pelas demais fonoaudiólogas envolvidas no Ambulatório de Fissura Labiopalatina, seguindo-se os mesmos critérios já descritos. Cada avaliadora não teve acesso às avaliações realizadas anteriormente e os resultados foram utilizados para determinar a confiabilidade entre os avaliadores.
Tabela 1. Análise de concordância entre juízes - Coeficiente Kappa

\begin{tabular}{clcc}
\hline Variável & K & P & $\begin{array}{c}\text { Intervalo de } \\
\text { confiança do } \\
\text { Kappa }\end{array}$ \\
\hline Hipernasalidade & 1,00 & $<0,001$ & $0,76-1,0$ \\
Grau de hipernasalidade & 0,730 & $<0,001$ & $0,56-0,90$ \\
Hiponasalidade & 1,00 & $<0,001$ & $0,76-1,0$ \\
Grau de hiponasalidade & 0,573 & $<0,001$ & $0,41-0,74$ \\
Inteligibilidade de fala & 1,00 & $<0,001$ & $0,76-1,0$ \\
Grau de inteligibilidade & 0,909 & $<0,001$ & $0,75-1,0$ \\
de fala & & &
\end{tabular}

Legenda: $\mathrm{K}$ = valor do coeficiente do teste Kappa, $\mathrm{P}=$ valor de significância estatística

Na Tabela 1, encontra-se descrita a análise dos resultados emitida independentemente pelos três fonoaudiólogos responsáveis pelos atendimentos, referentes à ressonância e inteligibilidade da fala. A comparação entre os juízes indicou alta concordância para todas as variáveis consideradas, confirmando que a análise é confiável. A análise de concordância entre os juízes foi calculada pelo coeficiente Kappa.

\section{Análise dos dados}

Os dados obtidos foram submetidos à análise estatística no software SPSS versão 22. A análise descritiva foi realizada pela frequência e porcentagem de distribuição para as variáveis categóricas, pela média, desvio padrão, valores mínimo e máximo, mediana e quartis para as variáveis numéricas. A análise inferencial foi empreendida pelo teste não paramétrico de Mann-Whitney para as comparações interruptos de variáveis numéricas, pelo teste Qui-quadrado ou pelo teste exato de Fisher para as variáveis categóricas. Para todas as comparações foi utilizado o nível de significância de 5\%.

\section{RESULTADOS}

\section{Caracterização geral dos participantes}

A Tabela 2 mostra a idade dos participantes dos GP e GT no momento da realização da palatoplastia e da avaliação fonoaudiológica. Foi possível observar que há um número maior de participantes que realizaram a palatoplastia tardiamente. Além disso, existe uma ampla variação da idade para a realização do procedimento cirúrgico, com realização da palatoplastia também na idade adulta.

Para a confirmação da diferença de idade à submissão da palatoplastia primária, comparou-se a mediana de idade dos participantes de ambos os grupos no momento da cirurgia e no momento da avaliação fonoaudiológica. Em ambas as comparações, os participantes do GT apresentaram maior mediana de idade, com diferença estatisticamente significante.

Foi realizada a análise do gênero dos participantes em relação aos diferentes grupos e sua verificação não evidenciou diferença estatística (Tabela 3). A verificação do tipo de fissura nos grupos não evidenciou diferença estatística, sendo que, em ambos os grupos, houve mais casos de fissura labiopalatina (Tabela 4). 


\section{Caracterização da fala dos participantes - análise geral e de grupos}

A classificação da ressonância (Tabela 5), de acordo com o GP e GT, mostra que para este parâmetro a classificação alterada esteve presente na maioria dos participantes. A avaliação fonoaudiológica relacionada à produção de fala não apontou diferença estatística entre os grupos para a classificação da ressonância, porém o valor de $\mathrm{p}$ foi marginal (próximo a 0,05 ), $\mathrm{o}$ que pode ser interpretado na Bioestatística como uma tendência. Se considerarmos tal tendência, é possível afirmar que o GT teria mais participantes com ressonância classificada como alterada.

Quando a análise é realizada de acordo com os tipos de alteração da ressonância, em ambos os grupos, é observado um maior número de participantes com hipernasalidade (Tabela 6).
A hiponasalidade só esteve presente em quatro participantes do GT e quando ocorreu foi em grau leve.

Para esta variável Hipernasalidade, não houve diferença entre os grupos quando considerado se estavam ausentes ou presentes, porém, ao verificar a distribuição de frequência de cada grau de alteração, pode-se perceber que no GP a porcentagem de participantes com alteração leve é maior do que no GT, enquanto no GT a porcentagem de participantes com alteração grave é maior que no GP (Tabela 7).

Não houve diferença entre os grupos para os parâmetros ronco nasal, fraca pressão intraoral e emissão de ar nasal audível (Tabela 8).

Com relação à inteligibilidade de fala, é possível observar que não houve diferença estatística entre os grupos, sendo que a maioria dos participantes apresentou inteligibilidade de fala

Tabela 2. Idade dos participantes do grupo precoce e tardio no momento de realização da palatoplastia e da avaliação fonoaudiológica

\begin{tabular}{cccccccccccc}
\hline Idade & Grupo & $\mathrm{n}$ & Média & Desvio-padrão & Mín & Máx & Med & $1^{\circ}$ quartil & $3^{\circ}$ quartil & Comparação \\
\hline Palatoplastia & $\mathrm{P}$ & 43 & 1,4 & 0,459 & 1 & 2 & 1,3 & 1,0 & 2,0 & $\mathrm{U}=0,0 \mathrm{z}=-8,472 \mathrm{p}<0,001^{*}$ \\
& $\mathrm{~T}$ & 54 & 11,5 & 10,69 & 3 & 50 & 7,0 & 4,0 & 15,8 & \\
Atual & $\mathrm{P}$ & 43 & 12,3 & 6,95 & 6 & 29 & 10,0 & 6,0 & 18,0 & $\mathrm{U}=724,0 \mathrm{z}=-3,183 \mathrm{p}=0,001^{*}$ \\
& $\mathrm{~T}$ & 54 & 19,8 & 12,37 & 6 & 50 & 16,0 & 9,0 & 29,3 & & \\
\hline
\end{tabular}

*diferença estatística $(p<0,05)$

Legenda: $\mathrm{n}$ = número de participantes; mín = idade mínima; máx = idade máxima; med = mediana; $\mathrm{P}=$ grupo precoce; $\mathrm{T}=$ grupo tardio

Tabela 3. Comparação do gênero dos participantes de cada grupo

\begin{tabular}{|c|c|c|c|c|}
\hline \multirow{2}{*}{ Grupo } & \multicolumn{2}{|c|}{ Gênero } & \multirow{2}{*}{ Total } & \multirow{2}{*}{$\mathrm{P}$} \\
\hline & Feminino & Masculino & & \\
\hline Precoce & 19 & 24 & 43 & $\mathrm{X}^{2}=0,867 \mathrm{gl}=1 \mathrm{p}=0,352^{*}$ \\
\hline Tardio & 29 & 25 & 54 & \\
\hline
\end{tabular}

*diferença estatística $(p<0,05)$ - Teste Qui-quadrado

Tabela 4. Comparação do tipo de fissura entre os grupos

\begin{tabular}{|c|c|c|c|c|}
\hline \multirow{2}{*}{ Tipo de fissura } & \multicolumn{2}{|c|}{ Grupo } & \multirow{2}{*}{ Total } & \multirow{2}{*}{ Estatística } \\
\hline & Precoce & Tardio & & \\
\hline Labiopalatal & 30 & 31 & 61 & $X^{2}=1,567 \mathrm{gl}=1 \mathrm{p}=0,211^{*}$ \\
\hline Palatal & 13 & 23 & 36 & \\
\hline
\end{tabular}

*diferença estatística $(p<0,05)$ - Teste Qui-quadrado

Tabela 5. Classificação da ressonância

\begin{tabular}{|c|c|c|c|c|c|c|}
\hline \multirow{2}{*}{$\begin{array}{c}\text { Classificação da } \\
\text { ressonância }\end{array}$} & \multicolumn{2}{|c|}{ GP } & \multicolumn{2}{|c|}{ GT } & \multirow{2}{*}{ Total } & \multirow{2}{*}{ Estatística } \\
\hline & Frequência & $\%$ & Frequência & $\%$ & & \\
\hline Adequada & 13 & 30,2 & 8 & 14,8 & 21 & $X^{2}=3,355 \mathrm{gl}=1 \mathrm{p}=0,067^{\star}$ \\
\hline Alterada & 30 & 69,8 & 46 & 85,2 & 76 & \\
\hline
\end{tabular}

*diferença estatística $(p<0,05)$ - Teste Qui-quadrado

Legenda: GP = grupo precoce; $\mathrm{GT}=$ grupo tardio

Tabela 6. Caracterização da hipernasalidade

\begin{tabular}{|c|c|c|c|c|c|c|c|}
\hline \multirow{2}{*}{$\begin{array}{l}\text { Alteração de } \\
\text { ressonância }\end{array}$} & \multirow{2}{*}{ Classificação } & \multicolumn{2}{|c|}{ GP } & \multicolumn{2}{|c|}{ GT } & \multirow{2}{*}{ Total } & \multirow{2}{*}{ Estatística } \\
\hline & & Frequência & $\%$ & Frequência & $\%$ & & \\
\hline \multirow[t]{2}{*}{ Hipernasalidade } & Ausente & 12 & 27,9 & 8 & 14,8 & 20 & $X^{2}=2,507 \mathrm{gl}=1 \mathrm{p}=0,113$ \\
\hline & Presente & 31 & 72,1 & 46 & 85,2 & 77 & \\
\hline \multirow[t]{2}{*}{ Hiponasalidade } & Ausente & 43 & 100,00 & 50 & 92,6 & 93 & $P=0,127$ \\
\hline & Presente (leve) & 0 & 0,0 & 4 & 7,4 & 4 & \\
\hline
\end{tabular}

Legenda: $\mathrm{GP}=$ grupo precoce; $\mathrm{GT}$ = grupo tardio 
alterada (Tabela 9), independentemente da idade da realização da palatoplastia. Porém, quando a análise é feita de acordo com os graus de alteração, como mostra a Tabela 10, no GT, é observado um grau maior de alteração de inteligibilidade de fala, sendo que, no GP, a porcentagem de participantes com alteração leve é maior do que no GT, enquanto, no GT, a porcentagem de participantes com alteração grave é maior que no GP.

A Tabela 11 apresenta a caracterização geral dos participantes quanto aos DACs. Observou-se maior ocorrência de participantes apresentando a alteração no GT, sendo o tipo de DAC mais comum o Golpe de Glote, seguido pela Fricativa Faríngea
(Tabela 12). No GP, o Golpe de Glote também ocorreu com maior frequência dentre os demais.

Na Tabela 13, estão descritos o número de fonemas alterados para cada tipo de DAC em cada um dos grupos. Para o Golpe de glote, no GP, o número de fonemas alterados na fala dos participantes variou de um até 12 fonemas, o que indica a variabilidade do perfil dos participantes dos grupos. No GT, também para o Golpe de Glote, o número de fonemas alterados variou de um até 13. A Tabela 14 mostra que os grupos se diferenciaram de forma estatisticamente significante quanto ao número de fonemas alterados.

Tabela 7. Grau de hipernasalidade

\begin{tabular}{cccccc}
\hline \multirow{2}{*}{ Grau da alteração } & \multicolumn{2}{c}{ GP } & & \multicolumn{2}{c}{ GT } \\
\cline { 2 - 5 } \cline { 5 - 6 } & Frequência & Frequência & 15 & 32,6 \\
Leve & 15 & 48,4 & 20 & 43,5 \\
Moderado & 13 & 41,9 & 11 & 23,9 \\
Grave & 3 & 9,7 & & & 0 \\
\hline
\end{tabular}

Legenda: $\mathrm{GP}=$ grupo precoce; $\mathrm{GT}$ = grupo tardio

Tabela 8. Presença de ronco nasal

\begin{tabular}{|c|c|c|c|c|c|c|c|}
\hline \multirow{2}{*}{ Variável } & \multirow{2}{*}{ Classificação } & \multicolumn{2}{|c|}{ GP } & \multicolumn{2}{|c|}{ GT } & \multirow{2}{*}{ Total } & \multirow{2}{*}{ Estatística } \\
\hline & & Frequência & $\%$ & Frequência & $\%$ & & \\
\hline \multirow[t]{2}{*}{ Ronco nasal } & Ausente & 37 & 86,0 & 51 & 94,4 & 88 & $p=0,179$ \\
\hline & Presente & 6 & 14,0 & 3 & 5,6 & 9 & \\
\hline \multirow{2}{*}{$\begin{array}{c}\text { Fraca pressão } \\
\text { intraoral }\end{array}$} & Ausente & 30 & 69,8 & 30 & 55,6 & 60 & $X^{2}=2,049 \mathrm{gl}=1 \mathrm{p}=0,152$ \\
\hline & Presente & 13 & 30,2 & 24 & 44,4 & 37 & \\
\hline \multirow{2}{*}{$\begin{array}{l}\text { Emissão de ar } \\
\text { nasal audível }\end{array}$} & Ausente & 33 & 76,7 & 37 & 68,5 & 70 & $X^{2}=0,806 \mathrm{gl}=1 \mathrm{p}=0,369$ \\
\hline & Presente & 10 & 23,3 & 17 & 31,5 & 27 & \\
\hline
\end{tabular}

Legenda: GP= grupo precoce; $\mathrm{GT}$ = grupo tardio

Tabela 9. Classificação da Inteligibilidade de fala

\begin{tabular}{|c|c|c|c|c|c|c|}
\hline \multirow{2}{*}{$\begin{array}{c}\text { Classificação da } \\
\text { inteligibilidade de } \\
\text { fala }\end{array}$} & \multicolumn{2}{|c|}{ GP } & \multicolumn{2}{|c|}{ GT } & \multirow[b]{2}{*}{ Total } & \multirow{2}{*}{ Estatística } \\
\hline & Frequência & $\%$ & Frequência & $\%$ & & \\
\hline Adequado & 13 & 30,2 & 9 & 16,7 & 22 & $\mathrm{X}^{2}=2,512 \mathrm{gl}=1 \mathrm{p}=0,113$ \\
\hline Alterado & 30 & 69,8 & 45 & 83,3 & 75 & \\
\hline Total & 43 & 100,0 & 54 & 100,0 & 97 & \\
\hline
\end{tabular}

Legenda: GP= grupo precoce; GT = grupo tardio

Tabela 10. Grau da inteligibilidade de fala

\begin{tabular}{cccccc}
\hline \multirow{2}{*}{$\begin{array}{c}\text { Grau da alteração da } \\
\text { inteligibilidade }\end{array}$} & GP & & \multicolumn{2}{c}{ GT } \\
\cline { 2 - 5 } \cline { 5 - 6 } & Frequência & Frequência & 11 & 24,4 \\
Leve & 17 & 56,7 & 21 & 46,7 \\
Grave & 9 & 30,0 & 13 & 28,9 \\
Total & 4 & 13,3 & 45 & 100,0 \\
\hline
\end{tabular}

Legenda: $\mathrm{GP}=$ grupo precoce; $\mathrm{GT}$ = grupo tardio

Tabela 11. Classificação dos DACs

\begin{tabular}{|c|c|c|c|c|c|}
\hline \multirow{2}{*}{ DACs } & \multicolumn{2}{|c|}{ GP } & \multicolumn{2}{|c|}{ GT } & \multirow{2}{*}{ Total } \\
\hline & Frequência & $\%$ & Frequência & $\%$ & \\
\hline Presente & 24 & 55,8 & 41 & 75,9 & 65 \\
\hline Ausente & 19 & 44,2 & 13 & 24,1 & 32 \\
\hline Total & 43 & 100,0 & 54 & 100,0 & 97 \\
\hline
\end{tabular}

Legenda: DACs = Distúrbio Articulatório Compensatório; GP= grupo precoce; GT = grupo tardio 
Tabela 12. Caracterização dos distúrbios articulatórios compensatórios

\begin{tabular}{cccccccc}
\hline & \multicolumn{7}{c}{ Tipos de Distúrbios compensatórios articulatórios } \\
\cline { 2 - 7 } Grupo & GG & PDMP & PF & FF & FNP & FN \\
& $\mathrm{n}$ & $\mathrm{n}$ & $\mathrm{n}$ & $\mathrm{N}$ & $\mathrm{N}$ & $\mathrm{N}$ \\
\hline Precoce & 19 & 6 & 4 & 4 & 0 & 5 & $\mathrm{n}$ \\
Tardio & 35 & 5 & 4 & 11 & 2 & 4 \\
\hline
\end{tabular}

Legenda: $\mathrm{n}=$ número de indivíduos; GG = Golpe de Glote; PMDP = Plosiva Dorso Médio Palatal; PF = Plosiva Faríngea; FF = Fricativa Faríngea; FNP = Fricativa Nasal Posterior; FN = Fricativa Nasal; FV = Fricativa Velar

Tabela 13. Número de fonemas em que os distúrbios articulatórios compensatórios foram verificados, conforme a divisão dos grupos (n=26)

\begin{tabular}{|c|c|c|c|c|c|c|c|c|c|c|c|c|c|c|}
\hline \multirow{2}{*}{$\begin{array}{l}\text { Número de } \\
\text { fonemas }\end{array}$} & \multicolumn{2}{|c|}{$\begin{array}{c}\mathrm{GG} \\
\mathrm{N}\end{array}$} & \multicolumn{2}{|c|}{$\begin{array}{l}\text { PDMP } \\
\mathrm{N}\end{array}$} & \multicolumn{2}{|c|}{$\begin{array}{c}\mathrm{PF} \\
\mathrm{n}\end{array}$} & \multicolumn{2}{|c|}{$\begin{array}{l}\mathrm{FF} \\
\mathrm{N}\end{array}$} & \multicolumn{2}{|c|}{$\begin{array}{c}\text { FNP } \\
\mathrm{N}\end{array}$} & \multicolumn{2}{|c|}{$\begin{array}{c}\mathrm{FN} \\
\mathrm{N}\end{array}$} & \multicolumn{2}{|c|}{$\begin{array}{l}\mathrm{FV} \\
\mathrm{N}\end{array}$} \\
\hline & GP & GT & GP & GT & GP & GT & GP & $\mathrm{GT}$ & GP & GT & GP & GT & GP & GT \\
\hline 1 & 6 & 2 & 1 & 3 & 2 & 2 & 1 & 3 & 0 & 1 & 3 & 2 & 1 & 3 \\
\hline 2 & 3 & 10 & 4 & 1 & 1 & 0 & 1 & 3 & 0 & 0 & 1 & 1 & 1 & 2 \\
\hline 3 & 2 & 4 & 0 & 0 & 0 & 0 & 2 & 0 & 0 & 1 & 1 & 0 & 1 & 2 \\
\hline 4 & 3 & 5 & 1 & 1 & 1 & 0 & 0 & 3 & 0 & 0 & 0 & 1 & 0 & 1 \\
\hline 5 & 0 & 1 & 0 & 0 & 0 & 1 & 0 & 1 & 0 & 0 & 0 & 0 & 0 & 0 \\
\hline 6 & 2 & 5 & 0 & 0 & 0 & 1 & 0 & 1 & 0 & 0 & 0 & 0 & 0 & 0 \\
\hline 7 & 1 & 3 & 0 & 0 & 0 & 0 & 0 & 0 & 0 & 0 & 0 & 0 & 0 & 0 \\
\hline 8 & 0 & 1 & 0 & 0 & 0 & 0 & 0 & 0 & 0 & 0 & 0 & 0 & 0 & 0 \\
\hline 9 & 0 & 1 & 0 & 0 & 0 & 0 & 0 & 0 & 0 & 0 & 0 & 0 & 0 & 0 \\
\hline 10 & 1 & 0 & 0 & 0 & 0 & 0 & 0 & 0 & 0 & 0 & 0 & 0 & 0 & 0 \\
\hline 11 & 0 & 0 & 0 & 0 & 0 & 0 & 0 & 0 & 0 & 0 & 0 & 0 & 0 & 0 \\
\hline 12 & 1 & 1 & 0 & 0 & 0 & 0 & 0 & 0 & 0 & 0 & 0 & 0 & 0 & 0 \\
\hline 13 & 0 & 1 & 0 & 0 & 0 & 0 & 0 & 0 & 0 & 0 & 0 & 0 & 0 & 0 \\
\hline
\end{tabular}

Legenda: $\mathrm{n}$ = número de indivíduos; GG = Golpe de Glote; PMDP = Plosiva Dorso Médio Palatal; PF = Plosiva Faríngea; FF = Fricativa Faríngea; FNP = Fricativa

Nasal Posterior; FN = Fricativa Nasal; FV = Fricativa Velar

Tabela 14. Fonemas alterados com ocorrência de distúrbios articulatórios compensatórios

\begin{tabular}{ccccccccccc}
\hline Grupo & $\mathrm{N}$ & Média & Desvio-padrão & Mín & Máx & Med & $1^{\circ}$ quartil & $3^{\circ}$ quartil & Comparação \\
\hline P & 43 & 2,6 & 3,07 & 0 & 12 & 2,0 & 0,0 & 4,0 & $\mathrm{U}=847,5$ \\
$\mathrm{~T}$ & 54 & 4,4 & 3,92 & 0 & 14 & 4,0 & 0,8 & 7,3 & $\mathrm{z}=-2,323 \mathrm{p}=0,020^{*}$ \\
\hline
\end{tabular}

*diferença estatística $(p<0,05)$

Legenda: $\mathrm{n}=$ número de participantes; mín= idade mínima; máx= idade máxima; med=mediana; $\mathrm{P}=$ grupo precoce; $\mathrm{T}=$ grupo tardio

\section{DISCUSSÃO}

A idade média para a realização da palatoplastia no GP foi de 1,4 anos. Conforme apontado por estudo anteriores ${ }^{(5,7,10,11)}$, esta idade seria compatível à idade preconizada por protocolos internacionais e nacionais, que afirmam que a realização da palatoplastia nesta idade diminui as chances de o indivíduo apresentar alterações na fala em decorrência da fissura.

Na literatura, é bem estabelecido que o reparo cirúrgico da fissura palatina deve ser realizado precocemente, antes de dois anos de idade ${ }^{(5,11)}$, principalmente considerando a questão da fala ${ }^{(5)}$.

Para o GT, a idade média da realização da palatoplastia primária foi de 11,5 anos. Estudos ${ }^{(16,25)}$ apontam que, em países em desenvolvimento, a falta de informação e dificuldade em acessar o sistema de saúde são os principais indicadores de realização de cirurgias primárias em momentos tardios, sendo esta uma característica da população atendida no HCFMUSP, que, muitas vezes, não tem acesso ao atendimento conforme idade preconizada pelos protocolos da área.

Este estudo concorda com os achados da literatura ${ }^{(12)} \mathrm{em}$ relação ao gênero. Houve predomínio do gênero masculino em detrimento do feminino ${ }^{(12,15)}$, embora sem resultados estatisticamente significativos.

Quanto ao tipo de fissura, para este estudo, foram somente elencados pacientes com fissura de palato \pm lábio, sendo excluídos os pacientes com fissura de lábio pura. Desta forma, a comparação com os dados epidemiológicos verificados durante a realização deste estudo fica impossibilitada na população estudada.

Em relação à caracterização da fala, de forma geral, o estudo identificou um maior número de participantes dos grupos apresentando alteração nos parâmetros hipernasalidade, inteligibilidade de fala e presença de DACs. Para a presença de ronco nasal, fraca pressão intraoral e emissão de ar nasal 
audível, o número de alterações foi menor, embora ainda deva ser considerado.

De acordo com os resultados obtidos na presente pesquisa, é possível observar que, mesmo sem diferença estatística, os dados sugerem desempenho pior nos participantes que realizaram a cirurgia tardiamente. A presença de DACs foi aspecto que diferenciou os grupos, e houve uma tendência para que a hipernasalidade também ocorresse de forma diferente, assim como já fora citado na literatura ${ }^{(6,7,9,21)}$. Estas diferenças podem estar relacionadas com a dificuldade do fechamento velofaríngeo que ocorre frequentemente quando a palatoplastia é realizada tardiamente ${ }^{(5,6,11)}$.

Cabe ressaltar que este estudo não pôde controlar o tipo de cirurgia e a quantidade de cirurgiões envolvida nelas, pela natureza de admissão dos pacientes no serviço.

No que diz respeito à ressonância, observa-se que, em relação à hipernasalidade, há uma classificação em grau mais grave quando a abordagem cirúrgica é mais tardia. Esse aspecto se justificaria pelo fato de o fechamento do palato ocorrer numa idade avançada, na qual o modelo de fala alterado já estaria estabelecido, assim como características anatômicas desfavoráveis com maiores chances de instalação ${ }^{(6,13,26)}$. É possível também correlacionar essa relação com a ideia amplamente defendida na literaturar ${ }^{(7,9)}$ sobre a importância da realização da palatoplastia primária precocemente. Quanto à hiponasalidade, no presente estudo, observa-se apenas a ocorrência em quatro participantes, o que confirma que a alteração de ressonância mais presente na fala do paciente fissurado é a hipernasalidade ${ }^{(14)}$.

Demais características da fala, apontadas na literatura como menos comuns ${ }^{(14,17)}$, também foram assim identificadas neste estudo. Os dois grupos apresentaram baixa ocorrência de ronco nasal e, clinicamente, a literatura aponta que esta não é uma alteração frequentemente observada ${ }^{(6)}$. A fraca pressão intra-oral também não é a alteração mais comumente observada na avaliação clínica e, quando ocorre, de forma geral está relacionada com fonemas plosivos ${ }^{(2,6)}$. No estudo, sua ocorrência foi relativa, mas não atingiu $50 \%$ da amostra dos grupos. Esse resultado pode ser correlacionado à presença elevada de DACs na população estudada. A emissão nasal audível também se apresentou alterada em menos de $40 \%$ dos participantes, não apresentando diferenças estatísticas entre os grupos. Considera-se que esta também não é uma das alterações mais comuns na fala do paciente fissurado ${ }^{(6)}$.

Quanto aos DACs, é possível observar maior ocorrência na abordagem cirúrgica tardiamente. Este resultado vai ao encontro de achados da literatura ${ }^{(7,9)}$, e, por tais alterações na fala, a conduta mais indicada é a realização de fonoterapia ${ }^{(7,9,25)}$. De acordo com a literatura ${ }^{(6-9)}$, na impossibilidade de realizar o fonema no ponto articulatório adequado, o indivíduo faz uma compensação para executá-lo da maneira mais próxima possível da produção correta. Segundo Perry e Kuehn ${ }^{(9)}$, a ocorrência de DACs justifica a realização da fonoterapia, e os casos sem IVF justificam a realização do acompanhamento fonoaudiológico periodicamente para o monitoramento da fala e a prevenção de alterações articulatórias. Os DACs, junto com a hipernasalidade, são as alterações mais comuns na fala dos pacientes fissurados ${ }^{(14,18)}$.

Outra alteração comumente presente na fala de fissurados é o prejuízo de sua inteligibilidade ${ }^{(5,6,11)}$. Assim, como na literatura, observou-se grande ocorrência nos participantes do estudo, principalmente em maior gravidade no GT. De acordo com os estudos anteriores ${ }^{(14,18)}$ o prejuízo na inteligibilidade de fala pode estar também associado à presença de DACs, concordando com os resultados deste estudo.

Um dos fatores que podem ser relacionados ao fato de haver maior número de abordagem cirúrgica tardia na população atendida no HCFMUSP é a falta de acesso à informação e aos centros de referência de atendimento aos pacientes fissurados ${ }^{(17)}$. A literatura ${ }^{(2,15-17)}$ refere esta dificuldade e cita que, em países em desenvolvimento, também se verifica a dificuldade de registro desta população. Esses aspectos associam-se à procura tardia para o tratamento. Muitos centros brasileiros apresentam o mesmo perfil do HCFMUSP com o recebimento de pacientes com falta de informação sobre a fissura e suas relações genéticas e os tratamentos necessários. Centros de referência internacionais ${ }^{(15,16,26)}$ apontam algumas propostas para a minimização deste problema, como a conscientização da população dos fatores de risco e ocorrência da fissura e a utilização de protocolos de tratamento padronizados ${ }^{(15,27)}$. Estudo nacional ${ }^{(28)}$ comparou os resultados de um mesmo serviço em dois momentos distintos e chegou à conclusão de que a padronização do atendimento dos pacientes portadores de fissura mediante aplicação de protocolos mostrou melhora nos resultados encontrados, mesmo em um curto período de tempo.

É possível observar diferença no acesso às informações, ao tratamento e adesão nos países desenvolvidos. De acordo com a literatura, em países como Inglaterra ${ }^{(12)}$ e $\mathrm{EUA}^{(29)}$, a cirurgia de palatoplastia primária ocorre até 1 ano de idade, o que minimiza os efeitos da fissura na fala. Em nosso serviço, muitos pacientes só têm acesso a ele quando adultos.

Estudos padronizados, controlados e randomizados sobre os aspectos da fala de indivíduos com fissura labiopalatina são escassos. Não são identificados na literatura valores preditivos de prevalência e incidência das alterações de fala, e os resultados encontrados são variáveis. Com este estudo, espera-se obter material para formulação de estudos mais bem estruturados e com maior evidência científica. Também possibilitará a discussão de protocolos clínicos, que auxiliarão diretamente a população estudada. A determinação de diretrizes clínicas baseadas em evidências e a de indicadores do serviço seriam o ponto alto da atividade fonoaudiológica após a compilação destes dados iniciais.

Acredita-se que a determinação do perfil dos pacientes atendidos no referido hospital auxiliará a reflexão sobre a assistência fonoaudiológica, assim como a discussão sobre as melhores terapêuticas, uni ou transdisciplinares, que poderão ser oferecidas ao paciente fissurado. A determinação do perfil da população atendida no hospital poderá auxiliar a organização do atendimento fonoaudiológico prestado, contribuindo para 
a organização e planejamento de um atendimento baseado em evidências e pautado nas premissas do conceito de avaliação tecnológica em saúde.

\section{CONCLUSÃO}

Diante do apresentado, foi possível caracterizar o perfil geral e de fala dos pacientes submetidos à palatoplastia primária do referido hospital escola, referência de atendimento a pacientes fissurados na cidade de São Paulo. Observou-se, de forma geral, prejuízo na fala dos participantes do estudo, sendo evidenciada diferença estatisticamente significante entre os resultados do procedimento precoce e tardio para a presença de DACs. A variável ressonância apresentou resultados estatísticos marginais, indicando tendência à diferenciação dos grupos também em relação a essa alteração. Concluiu-se que a realização da cirurgia precocemente traz melhores resultados em relação à fala.

\section{REFERÊNCIAS}

1. Freitas JAS, Neves LT, Almeida ALPF, Garib DG, Trindade-Suedam IK, Yaedú RYF, et al. Rehabilitative treatment of cleft lip and palate: experience of the Hospital for Rehabilitation of Craniofacial Anomalies/ USP (HRAC/USP) Part 1: overall aspects. J Appl Oral Sci. 2012;20(1):9-15. PMid:22437671. http://dx.doi.org/10.1590/S1678-77572012000100003.

2. Souza J, Raskin S. Estudo clínico e epidemiológico de fissuras orofaciais. J Pediatr. 2013;89(2):137-44. PMid:23642423. http://dx.doi.org/10.1016/j. jped.2013.03.010.

3. Cox TC. Taking it to the max: the genetic and developmental mechanisms coordinating midfacial morphogenesis and dysmorphology. Clin Genet. 2004;65(3):163-76. PMid:14756664. http://dx.doi.org/10.1111/j.00099163.2004.00225.x.

4. Nahai FR, Williams JK, Burstein FD, Martin J, Thomas J. The management of cleft lip and palate: pathways for treatment and longitudinal assessment. Semin Plast Surg. 2005;19(4):275-85. http:// dx.doi.org/10.1055/s-2005-925900.

5. Rohrich RJ, Love EJ, Byrd HS, Johns DF. Optimal timing of cleft palate closure. Plast Reconstr Surg. 2000;106(2):413-21, quiz 422, discussion 423-5. PMid:10946942. http://dx.doi.org/10.1097/00006534-20000800000026 .

6. Palandi BBB, Guedes ZCF. Aspecto de fala de indivíduos com fissura palatina e labial, corrigida em diferentes idades. Rev CEFAC. 2011;13(1):816. http://dx.doi.org/10.1590/S1516-18462011005000012.

7. Abdel-Aziz M. Speech outcome after early repair of cleft soft palate using Furlow technique. Int J Pediatr Otorhinolaryngol. 2013;77(1):85-8. PMid:23116906. http://dx.doi.org/10.1016/j.ijporl.2012.09.038.

8. Peterson-Falzone SJ, Trost-Cardamone JE, Karnell MP, Hardin-Jones M. The clinican's guide to treating cleft palate speech. St. Louis: Mosby; 2006. Effects of cleft and non-cleft VPI on speech in older children. p. 17-39.

9. Perry JL, Kuehn DP. Magnetic resonance imaging and computer reconstruction of the velopharyngeal mechanism. J Craniofac Surg. 2009;20(2, Suppl 2):1739-46. PMid:19816343. http://dx.doi.org/10.1097/ SCS.0b013e3181b5cf46.

10. Hortis-Dzierzbicka M, Radkowska E, Fudalej P. Speech outcomes in 10-year-old children with complete unilateral cleft lip and palate after onestage lip and palate repair in the first year of life. J Plast Reconstr Aesthet Surg. 2012;65(2):175-81. PMid:21978731. http://dx.doi.org/10.1016/j. bjps.2011.09.015.
11. Chapman KL, Hardin-Jones MA, Goldstein JA, Halter KA, Havlik RJ, Schulte J. Timing of palatal surgery and speech outcome. Cleft Palate Craniofac J. 2008;45(3):297-308. PMid:18452355. http://dx.doi. org/10.1597/06-244.

12. Fitzsimons KJ, Copley LP, Deacon SA, van der Meulen JA. Hospital care of children with a cleft in England. Arch Dis Child. 2013;98(12):970-4. PMid:23968774. http://dx.doi.org/10.1136/archdischild-2013-304271.

13. Perry JL, Kuehn DP. Magnetic resonance imaging and computer reconstruction of the velopharyngeal mechanism. J Craniofac Surg. 2009;20(2, Suppl 2):1739-46. PMid:19816343. http://dx.doi.org/10.1097/ SCS.0b013e3181b5cf46.

14. Lima MRF, Leal FB, Araújo SVS, Matos EF, Di Ninno CQMS, Britto ATBO. Atendimento fonoaudiológico intensivo em pacientes operados de fissura labiopalatina: relato de casos. Rev Soc Bras Fonoaudiol. 2007;12(3):240-6. http://dx.doi.org/10.1590/S1516-80342007000300012.

15. Shapira Y, Haklai Z, Blum I, Shpack N, Amitai Y. Prevalence of non-syndromic orofacial clefts among jews and arabs, by type, site, gender and geography: a multi-center study in Israel. Isr Med Assoc J. 2014;16(2):759-63. PMid:25630204

16. Ajike SO, Adebola RA, Efunkoya A, Adeoye J, Akitoye O, Veror N. Epidemiology of adult cleft patients in North-western Nigeria: our experience. Ann Afr Med. 2013;12(1):11-5. PMid:23480989. http:// dx.doi.org/10.4103/1596-3519.108243.

17. Penido FA, Noronha RMS, Caetano KI, Jesus MSV, Di Ninno CQMS, Britto ATBO. Correlação entre os achados do teste de emissão de ar nasal e da nasofaringoscopia em pacientes com fissura labiopalatina operada. Rev Soc Bras Fonoaudiol. 2007;12(2):126-34. http://dx.doi.org/10.1590/ S1516-80342007000200010.

18. Prandini EL, Pegoraro-Krook MI, Dutka JCR, Marino VCC. Occurrence of consonant production errors in liquid phonemes in children with operated cleft lip and palate. J Appl Oral Sci. 2011;19(6):579-85. PMid:22230991. http://dx.doi.org/10.1590/S1678-77572011000600007.

19. Schuster M, Maier A, Bocklet T, Nkenke E, Holst A, Eysholdt U, et al. Automatically evaluated degree of intelligibility of children with different cleft type from preschool and elementary school measured by automatic speech recognition. Int J Pediatr Otorhinolaryngol. 2012;76(3):362-9. PMid:22236457. http://dx.doi.org/10.1016/j.ijporl.2011.12.010.

20. Kuehn D, Moller KT. Speech and language issues in the cleft palate population: the state of the art. Cleft Palate Craniofac J. 2000;37(4):348. http://dx.doi.org/10.1597/1545-1569(2000)037<0348:SALIIT>2.3.CO;2.

21. Mituuti CT, Piazentin-Penna SHA, Brandão GR, Bento-Gonçalves CGA. Caracterização da fala de indivíduos submetidos à palatoplastia primária. Rev Soc Bras Fonoaudiol. 2010;15(3):355-61. http://dx.doi.org/10.1590/ S1516-80342010000300008.

22. Andrade CRF, Befi-Lopes DM, Fernandes FDM, Wertzner HF. ABFW Teste de Linguagem Infantil nas áreas de fonologia, vocabulário, fluência e pragmática. São Paulo: Ed. Pró Fono; 2004.

23. Henningsson G, Kuehn DP, Sell D, Sweeney T, Trost-Cardamone JE, Whitehill TL. Universal parameters for reporting speech outcomes in individuals with cleft palate. Cleft Palate Craniofac J. 2008;45(1):1-17. PMid:18215095. http://dx.doi.org/10.1597/06-086.1.

24. Trindade IEK, Genaro KF, Yamashita RP, Miguel HC, Fukushiro AP. Proposal for velopharyngeal function rating in a speech perceptual assessment. Pro Fono. 2005;17(2):259-62. PMid:16909536. http://dx.doi. org/10.1590/S0104-56872005000200015.

25. Jajja MR, Gilani A, Cawasji ZF, Imran S, Khan MS, Hashmi SS, et al. Oral clefts: a review of the cases and our experience at a single institution. J Pak Med Assoc. 2013;63(9):1098-102. PMid:24601184.

26. Vogel AP, Ibrahim HM, Reilly S, Kilpatrick N. A comparative study of two acoustic measures of hipernasality. J Speech Lang Hear Res. 2009;52(6):1640-51. PMid:19951929. http://dx.doi.org/10.1044/10924388(2009/08-0161).

27. Owotade FJ, Ogundipe OK, Ugboko VI, Okoje VN, Olasoji HO, Makinde $\mathrm{ON}$, et al. Awareness, knowledge and attitude on cleft lip and palate among 
antenatal clinic attendees of tertiary hospitals in Nigeria. Niger J Clin Pract. 2014;17(1):6-9. PMid:24326798. http://dx.doi.org/10.4103/11193077.122822 .

28. Alonso N, Tanikawa DYS, Lima JE Jr, Ferreira MC. Avaliação comparativa e evolutiva dos protocolos de atendimento dos pacientes fissurados. Rev Bras Cir Plast. 2010;25(3):434-8. http://dx.doi.org/10.1590/S198351752010000300006.

29. Basseri B, Kianmahd BD, Roostaeian J, Kohan E, Wasson KL, Basseri $\mathrm{RB}$, et al. Current national incidence, trends, and health care resource utilization of cleft lip-cleft palate. Plast Reconstr Surg. 2011;127(3):125562. PMid:21364426. http://dx.doi.org/10.1097/PRS.0b013e3182043af6.

\section{Contribuição dos autores}

KIM, LDM, NA e CRFA foram responsáveis pela concepção e delineamento do estudo; KIM e LDM foram responsáveis pela coleta, análise e interpretação dos dados; KIM, LDM, CRFA auxiliaram na redação ou revisão do artigo de forma intelectualmente importante; NA, CRFA foram responsáveis pela aprovação final da versão a ser publicada. 